\title{
Addressing Disparities in Prenatal Care via Telehealth During COVID-19: Prenatal Satisfaction Survey in East Harlem
}

\author{
Itamar Futterman, MD ${ }^{1}$ Emily Rosenfeld, DO ${ }^{1}$ Miriam Toaff, MD, MS ${ }^{1}$ Taryn Boucher, BA ${ }^{2}$ \\ Samantha Golden-Espinal, BS ${ }^{2}$ Kamilah Evans, BS ${ }^{2}$ Camille A. Clare, MD, MPH ${ }^{10}$ \\ ${ }^{1}$ Department of Obstetrics and Gynecology, New York Medical \\ College, Valhalla, New York \\ ${ }^{2}$ New York Medical College, School of Medicine, Valhalla, New York \\ Address for correspondence Itamar Futterman, MD, Department of \\ Obstetrics and Gynecology, New York Medical College, New York City \\ Health + Hospitals/Metropolitan, 1901 First Avenue Room 4B5, \\ New York, NY 10029 (e-mail: itamar.futterman@wmchealth.org).
}

Am J Perinatol 2021;38:88-92.

Keywords

- telemedicine

- patient satisfaction

- SAPS

- COVID-19

- prenatal care

- Hispanic
Objective In the setting of an inner city, safety net hospital, patient satisfaction with prenatal care conducted via telehealth was compared with in-person visits at the height of the novel coronavirus disease 2019 (COVID-19) pandemic.

Study Design Through this cross-sectional study, patients were identified who received at least one televisit and one in-person visit during the COVID-19 pandemic. The Short Assessment of Patient Satisfaction (SAPS) survey was used to measure patient satisfaction. Surveys pertaining to in-person and televisits were conducted at the end of a telephone encounter, and overall satisfaction scores were documented. Patients were excluded if they received in-person or virtual care only and not both. The SAPS score correlated with the degree of patient satisfaction.

Results A total of 140 patients were identified who received both virtual and inperson prenatal care from March 1, 2020 to May 1, 2020. One hundred and four patients (74\%) agreed to be surveyed: 77 (74\%) self-identified as Hispanic and 56 (54\%) stated that their primary language was Spanish. The overall median satisfaction score for televisits and in-person visits was 20 (interquartile range [IQR]: 20, 25) and 24 (IQR: $22,26)(p=0.008, Z$ score $=2.651)$. In patients who self-identified as Hispanic or identified their primary language as Spanish, there was no statistically significant difference in their satisfaction scores.

Conclusion While there were lower scores in patient satisfaction for televisits in every category, there were no clinically significant differences since all medians were in the "satisfied" range. By lowering patient exposure to severe acute respiratory syndromecoronavirus-2 (SARS-CoV-2), especially for those at risk for reduced access to care and higher COVID-19 cases by zip code, telehealth allowed for appropriate continuation of satisfactory prenatal care with no impact on patient perceived satisfaction of care.

Key Points

- Telehealth allowed for continuation of satisfactory prenatal care in Hispanic patients.

- Hispanic patients are at risk for reduced access to care.

- Telehealth was a useful tool for achieving patient-perceived satisfactory care.

received

July 29,2020

accepted

September 16, 2020

published online

October 10, 2020 (c) 2020. Thieme. All rights reserved.

Thieme Medical Publishers, Inc.,

333 Seventh Avenue, 18th Floor,

New York, NY 10001, USA
DOI https://doi.org/

10.1055/s-0040-1718695.

ISSN 0735-1631. 
The novel coronavirus disease 2019 (COVID-19) pandemic had a significant impact on all communities in New York City (NYC) and has been linked to the deaths of more than 17,000 people living in the five boroughs to date. ${ }^{1}$ Of these cases, the Hispanic community has experienced the largest number of deaths, accounting for nearly $33 \%$ of all NYC confirmed deaths due to the virus with a total nearing 5,500 confirmed cases. ${ }^{1}$ Several public media channels and the Centers for Disease Control and Prevention (CDC) have elucidated the link between specific racial groups that have been profoundly affected by COVID-19 and reduced access to health care. ${ }^{2,3}$

During this time, the obstetrical community dealt with an unprecedented reality as providers were encouraged to limit in-person patient interactions. Social distancing forced prenatal providers to adopt telehealth to provide safe and effective care during the pandemic's peak months. ${ }^{4,5}$ The rapid adoption of telemedicine technology is part of a growing trend with an estimated $261 \%$ increase in utilization of telemedicine since 2015. ${ }^{6,7}$ Though obstetricians have used telehealth for years, it has mainly been used to deliver obstetrical care to specific patient populations, that is, patients with gestational diabetes, those in rural communities, and for the targeted enhancement of care for high-risk pregnancies. $^{8-12}$ Existing research suggests that the provision of obstetrical care via telemedicine is safe and yields similar obstetrical outcomes when compared with traditional models of care. ${ }^{13}$ Recently, the American College of Obstetricians and Gynecologists (ACOG) has encouraged providers to integrate telehealth into mainstream obstetrics and gynecology and stated that technology-enhanced health care opportunities can effectively supplement the current standard of care. ${ }^{14}$

Little is currently known on the degree of patient satisfaction with a prenatal care experience with telehealth. A literature review has demonstrated only three studies investigating the topic of patient satisfaction during the time of antenatal care; they have shown high satisfaction rates in low-risk patients who received telehealth-centered prenatal care. ${ }^{13,15,16}$ In this survey-based study, we examined patient satisfaction with the telehealth prenatal care services that were provided from one of the most heavily affected communities in the country during the COVID-19 pandemic.

\section{Materials and Methods}

A cross-sectional study comparing patient satisfaction from virtual and in-person prenatal care during the time of the COVID-19 pandemic was conducted. Institutional review board (IRB) approval was obtained (L-14250) from New York Medical College, NYC Health + Hospitals and NYC Health + Hospitals/Metropolitan. All patients who were identified received at least one virtual and one in-person prenatal care visit during the height of the COVID-19 pandemic from March 1 to May 1, 2020. Patients received care at low-risk and high-risk prenatal clinics and were monitored throughout the pregnancy by a physician or a midwife. A validated satisfaction survey, The Short Assessment of Patient Satisfaction (SAPS), was used. ${ }^{17}$ Though the SAPS survey had not been used in our practice before, the survey format was adapted to receive and analyze feedback on patient experience during the period mentioned above. Surveys were conducted over the phone, and overall satisfaction scores were documented and compared. Patients were called only once, and during that conversation were asked to respond to the survey questions twice. Each set of questions was given in a different order. In the firstround, questions were asked in order of 1 to 7 , while in the second set questions were asked in a random order. Patients were asked to think of their in-person visit first since the purpose of this study was to compare a new tool of delivering prenatal care to the existing standard of in person care. All patients had at least one telehealth visit and one inperson visit, the order in which those were completed was not tracked as a variable. Nevertheless, the order in which the survey was conducted asked the patient to think of their inperson visit first and then the telehealth visit.

Patients were excluded if they received in-person or virtual care only and not both. SAPS scores were reported as continuous variables from 1 to 28 . The SAPS score correlated with degree of patient satisfaction: 0 to $10=$ very dissatisfied, 11 to $18=$ dissatisfied, 19 to $26=$ satisfied, and 27 to $28=$ very satisfied.

Patient demographic information included the following: age, parity, provider type (physician or midwife), race, ethnicity, number of televisits, access to online personal medical record, primary language, comorbidities (diabetes in pregnancy, chronic hypertension, thyroid disease, and/or twin gestation), poor obstetrical history defined as history of preterm birth, history of preterm prelabor rupture of membranes or history of intrauterine fetal demise, and whether pregnancy was considered to be high risk or low risk.

MedCalc software was used for descriptive statistical analysis. ${ }^{18}$ A normality of data test was performed showing that the data were skewed in favor of "satisfied" overall (skewness was -1.233 and the kurtosis was 4.124). Since the data were not normally distributed, Mann-Whitney $U$-test was used to examine differences in median satisfaction scores. Statistically significant results were defined as outcomes with $p$-value of 0.05 or less.

\section{Results}

A total of 140 patients were identified who received both virtual and in-person prenatal care from March 1, to May 1, 2020. Patient demographics are further described in - Table 1. One hundred and four patients (74\%) agreed to be surveyed: 77 (74\%) self-identified as Hispanic and 56 (54\%) stated that their primary language was Spanish. The mean number of televisits was 1.8. In the telehealth group, the total number of "dissatisfied" and "satisfied" patients was 10 and 94, respectively. In the in-person group, the total number of "dissatisfied" and "satisfied" patients was 7 and 97, respectively (Chi-square calculation of $p=0.448$ ). The overall median satisfaction score for televisits and in-person visits was 20 (interquartile range [IQR]: 20, 25) and 24 (IQR: 22, 26; $p=0.008, Z$ score $=2.651$ ). 


\begin{tabular}{|c|c|c|}
\hline \multicolumn{2}{|l|}{ Characteristic } & $\begin{array}{l}(n=104) \\
x / 104(\%)^{a}\end{array}$ \\
\hline \multicolumn{2}{|l|}{ Age (y) } & $31.2 \pm 6.28$ \\
\hline \multicolumn{2}{|c|}{ Number of televisits } & $1.8 \pm 0.94$ \\
\hline \multicolumn{2}{|c|}{ Gestational age at televisits (wk) } & $28.9 \pm 7.43$ \\
\hline \multirow[t]{2}{*}{ Parity } & Nulliparous & $28(27)$ \\
\hline & Multiparous & $76(73)$ \\
\hline \multirow[t]{4}{*}{ Race } & Black & $14(13)$ \\
\hline & White & $10(9)$ \\
\hline & Asian & $0(0)$ \\
\hline & Not specified & $79(76)$ \\
\hline \multirow{2}{*}{ Language } & English & $48(46)$ \\
\hline & Other & $56(54)$ \\
\hline \multirow[t]{2}{*}{ Ethnicity } & Hispanic & $77(74)$ \\
\hline & Non-Hispanic & $27(26)$ \\
\hline \multirow[t]{2}{*}{ Provider } & Physician & $62(60)$ \\
\hline & Midwife & $42(40)$ \\
\hline \multirow[t]{2}{*}{ Level of care } & High risk & $29(28)$ \\
\hline & Low risk & $75(72)$ \\
\hline \multicolumn{2}{|c|}{ Prior cesarean delivery } & $18(17)$ \\
\hline \multicolumn{2}{|c|}{ Online access to personal medical record } & $37(35)$ \\
\hline \multirow[t]{5}{*}{ Comorbidities } & Chronic hypertension & $4(3.8)$ \\
\hline & Diabetes in pregnancy & $13(12.5)$ \\
\hline & Thyroid disease & $3(2.8)$ \\
\hline & Twin gestation & $2(1.9)$ \\
\hline & ${ }^{\mathrm{b}}$ Poor obstetrical history & $5(4.8)$ \\
\hline
\end{tabular}

${ }^{a}$ Continues data are recorded as means with standard deviation or percentage calculated out of $n=104$.

${ }^{b}$ Poor obstetrical history: history of preterm prelabor rupture of membranes, intrauterine fetal demise, and preterm birth.

In patients who self-identified as Hispanic or who identified their primary language as Spanish, there was no statistically significant difference in their satisfaction scores.

The following groups had statistically significant differences with lower rates of satisfaction from televisits when compared with in person care: those who were multiparous, English speaking, non-Hispanics, identified as "other" ethnicity, those receiving care from a midwife, and those who were considered to be low risk ( - Table 2 ). Though statistically significant, none of these categories were found to be clinically significant since the median for both televisits and in-person visits were in the SAPS score range of "satisfied."

Intergroup analysis (described in -Table 3 ) showed that when median satisfaction scores were compared within each demographic variable, there were no statistically significant or clinically significant differences between satisfaction from televisits and in person encounters.

\section{Discussion}

Access to health care refers to the degree to which people can obtain appropriate health care promptly. Hispanics face a variety of financial and nonfinancial barriers to receiving proper and timely health care. Degree of acculturation, language, and immigration status, all significantly affect access to care. ${ }^{19,20}$ Despite efforts to limit communication barriers in health care, it remains central to health care delivery and significantly affects patient-provider relationships and the quality of health care received. Studies have found that language barriers between providers and patients may result in a lack of understanding of medication side effects, provider instructions, increased use of the emergency department, and inadequate follow-up. ${ }^{21}$ Prior research has identified two key barriers to health care access. The first is health insurance coverage and the second is not having a constant source of care. While the first is addressed by government-provided health insurance when establishing prenatal care, a steady health care provider remains a substantial struggle for the Hispanic patient population. ${ }^{22}$ Having a usual source of health care reduces nonfinancial barriers to obtaining care, improves access to services, and increases the frequency of contacts with health care providers. In particular, having a usual source of care provides an entry point into a complicated health care system when care is needed. ${ }^{22}$ Hispanics are among those who experience the most severe and concentrated types of health disparities, much of which are due to a lack of timely access to appropriate health care. ${ }^{23}$ The Institute of Medicine's report on the quality of health identified illiteracy and distrust of technology as potential barriers to telemedicine in urban underserved settings, and it is important to assess community perceptions and satisfaction of this technology. ${ }^{24}$

This study demonstrates that among Hispanic patients who are at risk for decreased access to care, the use of telehealth for prenatal care was a useful clinical tool for achieving comparable patient-perceived satisfactory care. As seen in other studies, telehealth satisfaction scores were similar when compared with in-person visits. ${ }^{13,15,16}$ In agreement with previous statements made by ACOG, ${ }^{4,14}$ telehealth should continue as a tool to provide prenatal care, especially at times of social distancing and in attempts of limiting provider-patient face-to-face encounters at least until more data are available regarding the difference in pregnancy outcomes between those who were managed in person and those primarily managed via telehealth during the months of COVID-19.

\section{Strengths and Limitations}

Our study's strengths were that it targeted a homogenous patient population with nearly $75 \%$ of the participants identifying as Hispanic and 54\% stating that their primary language was Spanish. We were able to reach 75\% (104/140) of patients who met the inclusion criteria.

The main limitation of this study was that there was a delay between the time the survey was conducted, and the last prenatal visit that patient had. None of the surveys were 


\begin{tabular}{|c|c|c|c|c|c|}
\hline \multicolumn{2}{|l|}{ Variable } & \multirow{2}{*}{$\begin{array}{l}\text { Median SAPS score telehealth } \\
23(22,26)\end{array}$} & \multirow{2}{*}{$\begin{array}{l}\text { Median SAPS score in person } \\
25(22,27)\end{array}$} & \multirow{2}{*}{$\begin{array}{l}Z \text { score } \\
-1.221\end{array}$} & \multirow{2}{*}{$\begin{array}{l}p \text {-Value }^{\mathrm{a}} \\
0.222\end{array}$} \\
\hline Parity & Nulliparous & & & & \\
\hline & Multiparous & $22(20,25)$ & $23(21.25,26)$ & 2.480 & 0.013 \\
\hline \multirow[t]{3}{*}{ Race } & African American & $22(21.75,25.25)$ & $25(20.75,27)$ & 0.896 & 0.368 \\
\hline & White & $21.5(16.75,25.5)$ & $25.5(21.75,26.25)$ & 1.361 & 0.174 \\
\hline & Other & $23(20,25)$ & $22(22,26)$ & 2.132 & 0.033 \\
\hline \multirow[t]{2}{*}{ Language } & English & $22(20.25,26)$ & $25(22,27)$ & 2.026 & 0.042 \\
\hline & Other & $23(20,25)$ & $23(21.5,25)$ & 1.641 & 0.101 \\
\hline \multirow[t]{2}{*}{ Ethnicity } & Hispanic & $23(20,25)$ & $23(21.5,25.5)$ & 1.742 & 0.082 \\
\hline & Non-Hispanic & $22(21,25)$ & $25.5(22,27)$ & 2.335 & 0.019 \\
\hline \multirow[t]{2}{*}{ Provider } & Physician & $23(21,25)$ & $24(22,26)$ & 1.334 & 0.184 \\
\hline & Midwife & $22(20,25)$ & $22(20,24.5)$ & -2.500 & 0.012 \\
\hline \multirow[t]{2}{*}{ Level of care } & High risk & $22(20,24.5)$ & $22(21,25)$ & 0.863 & 0.389 \\
\hline & Low risk & $23(20.25,25)$ & $24(22,27)$ & 2.599 & 0.009 \\
\hline \multicolumn{2}{|c|}{ Prior cesarean delivery } & $22(20,26.25)$ & $22.5(21,26.25)$ & 0.490 & 0.624 \\
\hline \multicolumn{2}{|c|}{$\begin{array}{l}\text { Access to online electronic medi- } \\
\text { cal record }\end{array}$} & $22.5(21,25)$ & $23(20,23.75)$ & 0.529 & 0.596 \\
\hline
\end{tabular}

Abbreviation: SAPS, short assessment of patient satisfaction.

${ }^{a} p$-Values calculated using Mann-Whitney U-test for continuous data that are not normally distributed, to compare medians (interquartile range) with $p<0.05$ to be considered statistically significant.

\begin{tabular}{|c|c|c|c|c|c|}
\hline \multirow[t]{2}{*}{ Variable } & & \multicolumn{4}{|c|}{ Telehealth SAPS score } \\
\hline & & \multicolumn{2}{|c|}{ Median SAPS score } & \multirow{2}{*}{$\begin{array}{l}Z \text { score } \\
-1.74\end{array}$} & \multirow{2}{*}{$\begin{array}{l}p \text {-Value }{ }^{\mathrm{a}} \\
0.082\end{array}$} \\
\hline Parity & multiparous versus nulliparous & $22(20,25)$ & $23(22,26)$ & & \\
\hline \multirow[t]{3}{*}{ Race } & African American versus all & $22(21.75,25.25)$ & $22(20,25)$ & -0.359 & 0.718 \\
\hline & Caucasian versus all & $21.5(16.75,25.5)$ & $22(20,25)$ & 0.744 & 0.459 \\
\hline & Other versus all & $23(20,25)$ & $22(20,25)$ & 0.053 & 0.960 \\
\hline Language & English versus non-English speakers & $22(20.25,26)$ & $23(20,25)$ & -0.389 & 0.687 \\
\hline Ethnicity & Hispanic versus non-Hispanic & $23(20,25)$ & $22(21,25)$ & 0.156 & 0.873 \\
\hline Provider & Physician versus midwife & $23(21,25)$ & $22(20,25)$ & 1.419 & 0.156 \\
\hline Level of care & High risk versus low risk & $22(20,24.5)$ & $23(20.25,25)$ & 1.598 & 0.109 \\
\hline $\begin{array}{l}\text { Prior cesarean } \\
\text { delivery }\end{array}$ & Yes versus no & $22(20,26.25)$ & $22(20,25)$ & -0.076 & 0.936 \\
\hline $\begin{array}{l}\text { Access to online } \\
\text { electronic medical record }\end{array}$ & Yes versus no & $22.5(21,25)$ & $22(20,25)$ & 0.023 & 0.984 \\
\hline
\end{tabular}

Abbreviation: SAPS, short assessment of patient satisfaction.

${ }^{a} p$-Values calculated using Mann-Whitney U-test for continuous data that are not normally distributed to compare medians (interquartile range) with $p<0.05$ to be considered statistically significant.

administered at the time of a prenatal appointment. While this could introduce recall bias, we attempted to eliminate this bias by conducting the survey no more than 5 days after the last prenatal encounter.

\section{Conclusion}

By lowering patient exposure to SARS-CoV-2, especially for those at risk for reduced access to care and higher COVID-19 cases by zip code, as published by the $\mathrm{CDC},{ }^{25,26}$ telehealth allowed for an appropriate continuation of adequate prenatal care with no impact on patient-perceived satisfaction of care. While the impact on access to care continues to unravel, telehealth will likely continue to address issues like constant care providers, shorter wait times in the waiting room of a given clinic or provider's office, and will continue to serve as a solution for social distancing. As seen in this study, Hispanic patients with risks for 
reduced access to care perceive telehealth as a suitable alternative for in person encounters.

\section{Conflict of Interest}

None declared.

\section{References}

1 Details on deaths. Available at: https://www1.nyc.gov/site/doh/ covid/covid-19-data-deaths.page. Accessed June 28, 2020

2 Turner-Musa J, Ajayi O, Kemp L. Examining social determinants of health, stigma, and COVID-19 disparities. Healthcare (Basel) 2020;8(02):168

3 Abuelgasim E, Saw LJ, Shirke M, Zeinah M, Harky A. COVID-19: unique public health issues facing Black, Asian and minority ethnic communities. Curr Probl Cardiol 2020;45(08):100621

4 COVID-19 FAQs for obstetrician-gynecologists, telehealth. Available at: https://www.acog.org/clinical-information/physicianfaqs/covid-19-faqs-for-ob-gyns-telehealth. Accessed September 25, 2020

5 Novel coronavirus "COVID-19": special considerations for pregnant women. Available at: https://www.kff.org/coronaviruscovid-19/issue-brief/novel-coronavirus-covid-19-special-considerations-for-pregnant-women/. Accessed June 28, 2020

6 Telemedicine is poised to grow as its popularity increases among physicians and patients. Available at: https://www.healthcarefinancenews.com/news/telemedicine-poised-grow-its-popularity-increases-among-physicians-and-patients. Accessed April 24, 2020

7 COVID-19 may permanently alter the telehealth landscape, from reimbursement to utilization. Available at: https://www.healthcarefinancenews.com/node/140009. Accessed April 24, 2020

8 Long MC, Angtuaco T, Lowery C. Ultrasound in telemedicine: its impact in high-risk obstetric health care delivery. Ultrasound $\mathrm{Q}$ 2014;30(03):167-172

9 Lowery C, Bronstein J, McGhee J, Ott R, Reece EA, Mays GP. ANGELS and University of Arkansas for Medical Sciences paradigm for distant obstetrical care delivery. Am J Obstet Gynecol 2007;196 (06):534.e1-534.e9

10 Odibo IN, Wendel PJ, Magann EF. Telemedicine in obstetrics. Clin Obstet Gynecol 2013;56(03):422-433

11 Wood D. STORC helps deliver healthy babies: the telemedicine program that serves rural women with high-risk pregnancies. Telemed J E Health 2011;17(01):2-4

12 Nudell J, Slade A, Jovanovič L, Hod M. Technology and pregnancy. Int J Clin Pract Suppl 2011;(170):55-60
13 Pflugeisen BM, McCarren C, Poore S, Carlile M, Schroeder R. Virtual visits: managing prenatal care with modern technology. MCN Am J Matern Child Nurs 2016;41(01):24-30

14 Implementing Telehealth in Practice. Implementing telehealth in practice. Obstet Gynecol 2020;135(02):e73-e79

15 Marko KI, Ganju N, Brown J, Benham J, Gaba ND. Remote prenatal care monitoring with digital health tools can reduce visit frequency while improving satisfaction. Obstet Gynec 2016;127 (p1S)

16 McDuffie RS Jr, Beck A, Bischoff K, Cross J, Orleans M. Effect of frequency of prenatal care visits on perinatal outcome among low-risk women. A randomized controlled trial. JAMA 1996;275 (11):847-851

17 Hawthorne G, Sansoni J, Hayes L, Marosszeky N, Sansoni E. Measuring patient satisfaction with health care treatment using the Short Assessment of Patient Satisfaction measure delivered superior and robust satisfaction estimates. J Clin Epidemiol 2014; 67(05):527-537

18 Schoonjans F. MedCalc statistical software. . Available at: https:// www.medcalc.org/index.php. Accessed June 28, 2020

19 Wells KB, Golding JM, Hough RL, Burnam MA, Karno M. Acculturation and the probability of use of health services by Mexican Americans. Health Serv Res 1989;24(02):237-257

20 Escarce JJ, Kapur K. Access to and Quality of Health Care. In: Tienda M, Mitchell F, eds. Hispanics and the Future of America: National Research Council (US) Panel on Hispanics in the United States. Washington, DC: National Academies Press (U.S.); 2006

21 David RA, Rhee M. The impact of language as a barrier to effective health care in an underserved urban Hispanic community. Mt Sinai J Med 1998;65(5-6):393-397

22 Lewin-Epstein N. Determinants of regular source of health care in black, Mexican, Puerto Rican, and non-Hispanic white populations. Med Care 1991;29(06):543-557

23 Nelson A. Unequal treatment: confronting racial and ethnic disparities in health care. J Natl Med Assoc 2002;94(08):666-668

24 Leavitt M. Medscape's response to the Institute of Medicine Report: Crossing the quality chasm: a new health system for the 21st century. MedGenMed 2001;3(02):2

25 Centers for Disease Control and prevention Reduced access to care: household pulse survey. Available at: https://www.cdc.gov/ nchs/covid19/pulse/reduced-access-to-care.htm. Published 2020. Accessed June 28, 2020

26 Total count of COVID-19 cases based on patient address by ZIP code. Available at: https://www1.nyc.gov/assets/doh/downloads/pdf/ imm/covid-19-cases-by-zip-04032020-1.pdf. Accessed September 25, 2020 